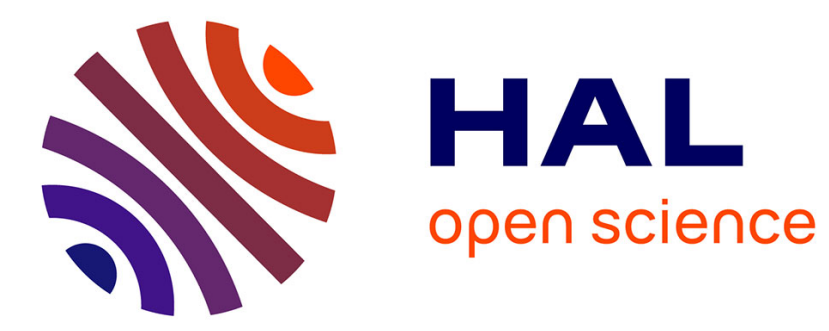

\title{
Variations de la taille corporelle chez les ongulés pléistocènes : implications paléoécologiques et taphonomiques
}

Pierre Magniez, Nicolas Boulbes, Jean-Philip Brugal

\section{- To cite this version:}

Pierre Magniez, Nicolas Boulbes, Jean-Philip Brugal. Variations de la taille corporelle chez les ongulés pléistocènes: implications paléoécologiques et taphonomiques. TaphonomieS, Editions des archives contemporaines, collection "Sciences archéologiques", 2017. hal-01744740

\author{
HAL Id: hal-01744740 \\ https://hal.science/hal-01744740
}

Submitted on 27 Mar 2018

HAL is a multi-disciplinary open access archive for the deposit and dissemination of scientific research documents, whether they are published or not. The documents may come from teaching and research institutions in France or abroad, or from public or private research centers.
L'archive ouverte pluridisciplinaire HAL, est destinée au dépôt et à la diffusion de documents scientifiques de niveau recherche, publiés ou non, émanant des établissements d'enseignement et de recherche français ou étrangers, des laboratoires publics ou privés. 


\title{
Variations de la taille corporelle chez les ongulés pléistocènes : implications paléoécologiques et taphonomiques
}

\author{
Pierre Magniez $^{1}$, Nicolas Boulbes ${ }^{2}$, Jean-Philip Brugal ${ }^{1}$ \\ ${ }^{1}$ UMR 7269 LAMPEA, Aix-en-Provence, Université Aix-Marseille - \\ CNRS - ministère de la Culture et de la Communication \\ ${ }^{2}$ UMR 7194 MNHN - UPVD - EPCC Centre européen \\ de recherches préhistoriques de Tautavel
}

\section{Introduction}

La taille corporelle des animaux est un paramètre très étudié en biologie comme en paléontologie car lié à leur physiologie et leur écologie. Elle est corrélée à d'autres paramètres majeurs, tels que la masse (poids), le taux de croissance, la fécondité ou le métabolisme [1-5]. De plus, il y a aussi des relations entre la taille et l'abondance des individus / espèces sur un territoire, leur aire potentielle de répartition et certains marqueurs sociaux [6-9].

De nombreux travaux traitent des tendances évolutives ou de la variabilité morphométrique des mammifères quaternaires en rapport avec les paléoenvironnements et/ou les paléoclimats, comme chez les carnivores [10-14], les cervidés [13, 15-18], les équidés [19-20] ou encore les bovidés [21-22].

L'estimation du gabarit des mammifères terrestres représentés dans les ensembles fossiles est donc très informative de plusieurs composantes de leur paléobiologie comme le degré de mobilité, leurs adaptations morphologiques (alimentation, locomotion) et acclimatations liées aux oscillations cycliques du climat au Pléistocène, tout autant que sur le degré des compétitions inter/intra spécifiques. En particulier, des relations ont été établies entre les variations de taille, la latitude et la saisonnalité, en accord ou non avec la règle de Bergmann [23]. Le climat peut affecter la taille au travers de changements écophénotypiques ou génétiques chez les mammifères au cours des cycles climatiques du Quaternaire [24-25]. Toutefois, déterminer des liens de causalité reste délicat, notamment pour distinguer ce qui a trait à la plasticité phénotypique plutôt 
qu'à des processus microévolutifs. En effet, les modèles (paléo)écologiques résultent d'interactions complexes entre les individus, les paramètres abiotiques des écosystèmes, la productivité végétale et la phénologie des plantes ainsi que des relations entre espèces pour l'accessibilité aux ressources. Nous avons choisi de développer ici trois exemples de variation chez des ongulés (cervidés, équidés, bovinés) bien représentés dans les ensembles osseux du Pléistocène et qui illustrent cette complexité. Ces données paléoécologiques ont également des implications sur les conditions taphonomiques de ces ensembles.

\section{Cas d'étude}

\subsection{Le renne (P. M.)}

Actuellement, le renne (Rangifer tarandus) présente une très vaste aire de distribution autour du cercle polaire, entre $46^{\circ}$ et $80^{\circ}$ de latitude nord et $5^{\circ}$ est à $14^{\circ}$ ouest. Plusieurs sous-espèces ont été différenciées suivant des traits anatomiques (au niveau du crâne notamment) et des caractéristiques externes telles que la forme des bois ou la couleur du pelage [26-27]. D'importantes variations de taille / masse sont enregistrées entre les sous-espèces en fonction des paramètres biotiques et abiotiques définissant leur environnement. Ainsi, la densité populationnelle des biomes parcourus, mais également la topographie des milieux (toundra, forêt, montagne) et le degré des déplacements ou migrations (courte / longue distance; latitudinale / altitudinale) sont des facteurs participant à la variation observée. De nombreux travaux pointent la grande capacité adaptative du renne et sa sensibilité aux modifications de son environnement se traduisant par une variabilité des phénotypes exprimés [27-32].

Les premières occurrences de renne en Europe occidentale sont attribuées à la première moitié du Pléistocène moyen, corrélées aux climats froids et secs. De rares vestiges ont été mis au jour en Europe septentrionale (Sussenbörn, Mosbach, Bad Frankenhausen, Westbury [33]) mais c'est en Europe méridionale que les plus importantes séries ont été découvertes, à la Caune de l'Arago (Pyrénées Orientales) dans des niveaux attribués aux stades isotopiques 14 et 12 (env. 0,6-0,4 Ma) avec plusieurs dizaines d'individus consommés par les Acheuléens. Au cours de la seconde moitié du Pléistocène moyen, le renne reste toujours faiblement représenté. En revanche, il est largement documenté en Europe au Pléistocène supérieur, pendant la dernière glaciation (env. 120 00010000 ans). Les fouilles de sites archéologiques du Paléolithique supérieur dénotent un statut socio-économique privilégié pour cette espèce (« âge du renne »).

L'étude diachronique de leur variation de taille corporelle est fondamentale puisqu'elle permet d'accéder à la fois à des informations liées à la structure des populations, à leur mobilité mais également aux caractéristiques paléoécologiques des milieux [16, 34-38]. Un modèle simple illustre cette variation, portant sur la hauteur latérale du talus (élément squelettique compact, abondant dans les sites archéo-paléontologiques et bon indicateur du gabarit de l'individu) de plusieurs sites du Pléistocène moyen et supérieur, majoritairement situés dans la moitié sud de la France (Figure 1). L'analyse des séries fossiles ne dégage pas de tendance linéaire mais enregistre des variations significatives. Globalement, les rennes du Pléistocène moyen ancien présentent un gabarit plus important par rapport aux séries du Pléistocène supérieur. Les différences 


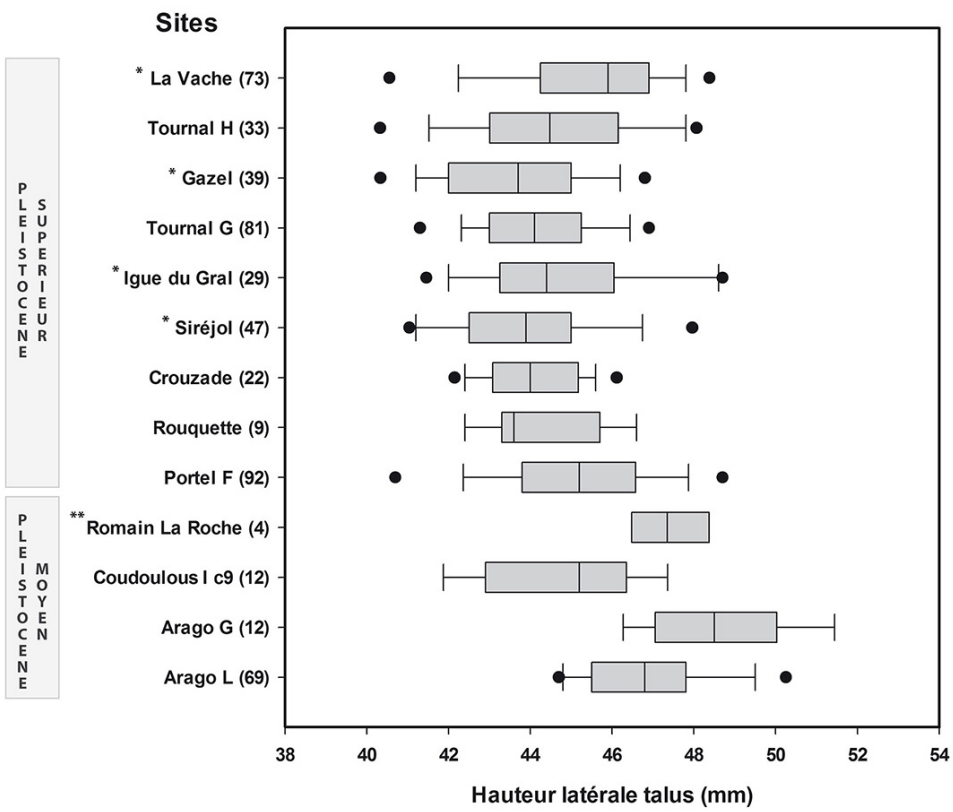

Figure 1 - Diagramme en boîte (médiane, quartiles, extrêmes) exprimant la variation de la hauteur latérale du talus de séries pléistocènes de renne ordonnées chronologiquement (le plus ancien en bas); * d'après [38] et ** d'après [39].

observées entre les deux niveaux de l'Arago peuvent traduire à la fois une variation de sex-ratio et/ou de conditions changeantes du milieu : froid et sec dans le niveau L et plus d'humidité dans le niveau G. Cela pourrait suggérer également des densités de populations relativement faibles sur des territoires nouvellement colonisés par l'espèce.

À la transition Pléistocène moyen / supérieur les séries de Coudoulous I (c. 9, Lot) et de Romain-la-Roche (Doubs) sont contrastées. Les différences pourraient tout-àfait s'expliquer ici par le sex-ratio : quatre éléments, très homogènes sur le plan des dimensions, seraient des mâles à Romain-la-Roche tandis que les rennes de Coudoulous I présentent une plus large distribution avec vraisemblablement une majorité de femelles.

$\mathrm{Au}$ Pléistocène supérieur deux groupes se dégagent : les sites du Portel-Ouest et de La Vache (Ariège), la grotte Tournal-niveau H (Aude) et l'Igue du Gral (Lot) présentent des rennes au gabarit plus important que ceux des sites de la Rouquette (Tarn), Siréjol (Lot), Tournal-niveau G, Gazel et la Crouzade (Aude). Il faut alors tenir compte de la représentativité de ces taphocœenoses, des structures biologiques représentées en rapport avec les contextes de formation des sites. Par exemple, le grand gabarit des rennes de La Vache s'explique par la forte proportion de mâles dans l'ensemble osseux [38]. Néanmoins, en synthétisant les résultats avec les informations d'autres disciplines connexes (ex. sédimentologie et palynologie), les séries de rennes du Pléistocène tardif du sud-ouest de la France suggèrent un changement spatio-temporel significatif de la 
taille corporelle en relation avec différentes conditions environnementales. Ainsi, les plus grands rennes du Tardiglaciaire sont contemporains d'une amélioration climatique, documentée dans les Pyrénées, qui annonce le Bølling (env. 14600 ans BP). Cette amélioration est marquée par une diminution des graminö̈des, une phase de renoncules (Ranunculus) et un développement de genévrier (Juniperus) et de pin (Pinus) [40].

Les oscillations climatiques influent indirectement sur les paramètres démographiques liés à la reproduction et la survie des individus (appelés également traits d'histoire de vie), d'autant plus que le renne est très sensible aux facteurs dépendants de la densité. Les extrêmes climatiques (aridité, températures) limitent sensiblement sa croissance. Le rôle clé est l'accessibilité et la sélection des fourrages saisonniers (i.e., phénologie des plantes et valeurs nutritionnelles) étroitement liées aux densités de population et à la ségrégation sexuelle. Les hivers rigoureux (trop froids et humides) entraînent une accessibilité limitée aux végétaux en raison de l'épaisseur du couvert neigeux et de possibles couches de glace au sol. Au contraire, lorsque la disponibilité végétale est favorable (en quantité et qualité), la compétition intraspécifique diminue et alors la taille corporelle tend à augmenter. Ce processus est prépondérant pour les femelles qui, à terme, conduit à une réduction du dimorphisme sexuel.

Cette variabilité de l'intensité du dimorphisme sexuel suivant les paramètres écologiques du milieu a été démontrée pour les populations actuelles de renne, et implique différentes stratégies de reproduction entre sexes. Chez les femelles; l'investissement dans la taille peut se produire après la maturité sous des conditions favorables, alors qu'elles investissent davantage dans la reproduction que dans la croissance corporelle lorsque les ressources fourragères sont limitées [30, 41-42]. Un autre cervidé, l'élan, montre un dimorphisme sexuel plus élevé dans les régions où la saison de croissance végétale est courte [43]. Sur le matériel de renne de la fin du Pléistocène supérieur dans le Sud-Ouest de la France, il semblerait que l'amplitude des variations de taille soit plus importante chez les femelles que chez les mâles [36], bien qu'il demeure parfois délicat de sexer à partir d'ossements. Si ce modèle est correct, le dimorphisme sexuel tendrait alors à diminuer dans cet espace-temps car soumis à de meilleures conditions environnementales, c'est-à-dire montrant une augmentation de l'humidité (et peut-être de la température) accompagnant une saison de croissance végétale plus longue. Toutefois, il est surprenant d'observer une relative stabilité parmi les mâles, alors qu'il devrait présenter un fort gabarit dans ces environnements productifs [27]. Ces données doivent donc être encore considérées avec prudence et nécessitent de nouveaux compléments méthodologiques (imagerie numérique 3D des ossements par exemple).

Aux variations diachroniques de la taille corporelle, il est également possible de documenter des variations d'ordre strictement spatiales en Europe (cline de taille). Suite à l'étude de matériel de sites archéologiques du Tardiglaciaire européen, des gradients NE-SO et E-W semblent pouvoir se différencier [16], comme le montrent d'autres travaux sur l'actuel [27-29]. En particulier, les données de séries fossiles rapportent une diminution de taille corporelle avec la latitude. Les rennes vivant dans le sud de leur aire de répartition montrent généralement une plus grande taille corporelle que 
leurs congénères des latitudes septentrionales. Cependant, sur une aire géographique plus restreinte (ex. Sud de la France) les variations ne semblent pas être significatives [38]. Enfin, une des difficultés - générale à toutes les espèces et associations fossiles est aussi d'obtenir une meilleure résolution chronologique afin de mieux déterminer la synchronicité ou les successions des séries et des sites.

La variation observée illustre plutôt la capacité rapide du génotype à produire des phénotypes préférentiels en réponse aux conditions des paléo-milieux, et désigne alors plus une certaine plasticité phénotypique qu'un mécanisme micro-évolutif. De fait, en améliorant certaines analyses (sexage des individus notamment), l'étude de la variabilité de la taille corporelle chez le renne pourrait être utilisée comme une approximation fiable des conditions environnementales (estimation de l'humidité / sécheresse ; amplitude / durée des saisons). L'approche morphométrique pourra à terme nous renseigner sur les écotypes et biotopes (toundra, forêt, montagne). Les rennes ont constitué un gibier préférentiel pour de nombreuses sociétés paléolithiques. Leurs variations de taille sont différentes selon les sexes et les environnements. Ces données ont un impact dans les modes d'acquisition de ce gibier, et donc dans la représentation des ensembles osseux, et dans les degrés d'exploitation (consommation, technologie) ou de conservation qui sont autant d'agents taphonomiques potentiels qu'il est nécessaire de considérer.

\subsection{Le cheval (N. B.)}

Le cheval est représenté aujourd'hui par une seule espèce à l'état sauvage, le cheval de Prjevalski, Equus przewalskii, éteint dans la nature mais perpétué dans les zoos et réintroduit dans différentes régions notamment en Mongolie. Les équidés caballins, arrivés en Europe au tout début du Pléistocène moyen, sont attestés par de nombreuses formes, espèces ou sous-espèces de tailles différentes. Les restes de chevaux sont très fréquents dans les sites paléolithiques. Leurs grandes capacités adaptatives squelettiques et dentaires assurent à ces derniers une présence sous des conditions climatiques et des environnements très variés, depuis les forêts interglaciaires jusqu'aux steppes / prairies pendant les épisodes glaciaires [44-47]. Contrairement à d'autres groupes d'ongulés (cervidés, bovinés), la taille chez les chevaux ne présente pas de dimorphisme sexuel [48-49].

De nombreux travaux portent sur les variations de la taille des chevaux au cours du Pléistocène [20, 46, 50-59]. Globalement, durant le Quaternaire, on observe une tendance évolutive vers une diminution de la taille. Celle-ci fluctue autour d'une grande moyenne jusqu'à la fin du Pléistocène moyen puis elle diminue sensiblement au Pléistocène supérieur, avant de s'accélérer à l'Holocène [55, 60]. Plusieurs exemples illustrent les modèles de variations de taille des chevaux à partir des séries pléistocènes de sites méditerranéens. La méthode du Variable Size Index (VSI [16-61]) permet de comparer les éléments du squelette postcrânien de ces séries par rapport à une série standard : site allemand de Mosbach pour le Pléistocène moyen et site français de Jaurens pour le Pléistocène supérieur. 


\subsubsection{Pléistocène moyen}

Dans la séquence du Complexe Moyen (CM) de la Caune de l'Arago (PyrénéesOrientales) attribuée au Pléistocène moyen médian, la taille du cheval fluctue avec une tendance générale à la diminution (Figure 2). Le gabarit est significativement plus fort chez les chevaux des niveaux inférieurs de l'ensemble I (CM I, niveaux Q-P) corrélés à l'épisode glaciaire du stade isotopique marin (SIM) 14 (env. 550000 ans), alors qu'il diminue nettement dans l'ensemble II (CM II, niveaux H-I-J) associés à l'interglaciaire du SIM 13 (env. 500000 ans); il augmente de nouveau sensiblement au cours de l'ensemble III (CM III, niveaux G- F, SIM 12, env. 450000 ans) sans atteindre la taille maximale des niveaux inférieurs (Figure 2). Enfin au cheval plus récent chronologiquement de l'interglaciaire de Lunel-Viel (Hérault) (SIM 9 ?, env. 330000 ans) correspond une taille significativement plus petite (Figure 2).

La forte taille du cheval des niveaux inférieurs (CM I) de la Caune de l'Arago est comparable au référentiel chronologiquement proche : le cheval de Mosbach (E. mosbachensis). Cette correspondance de gabarit peut s'expliquer par la contemporanéité des deux sites. Cependant le cheval de la Caune de l'Arago possède des caractères qui lui sont propres : museau plus court et large, macrodontie, extrémité des pattes plus robuste. Celui-ci a pu développer des adaptations particulières au milieu glaciaire en zone méditerranéenne en réponse au climat, en l'occurrence rigoureux, froid et sec, comme le suggèrent les autres proxys [62-68]. Le gabarit plus faible du cheval des niveaux du CM II par rapport aux niveaux du complexe inférieur (CM I) ou supérieur (CM III) parait lié aux conditions interglaciaires du SIM 13 (règle de Bergmann) plus tempérées et humides et à la fermeture du milieu mais les données dans ces niveaux restent pour le moment insuffisantes.

La diminution de taille (hautement significative $\mathrm{p}<0,001$ ) du cheval de Lunel-Viel, sous des conditions également interglaciaires, pourrait répondre au même phénomène et démontrer l'importance des climats. Son gabarit plus réduit, son museau plus allongé et ses extrémités des membres robustes ont été d'ailleurs interprétés comme une adaptation particulière en milieu tempéré humide [69-70].

Dans d'autres régions, notamment en Europe centrale (Allemagne), la comparaison entre les VSI et les rapports des isotopes de l'oxygène $\left({ }^{18} \mathrm{O} /{ }^{16} \mathrm{O}\right)$ des tissus osseux [72] tend à montrer que les grands chevaux sont plutôt associés aux températures élevées [57]. Selon Van Asperen [46-73], les habitats en mosaïque de certains interglaciaires continentaux aux conditions relativement sèches et à la couverture végétale diversifiée, sont très favorables aux chevaux caractérisés par une grande taille et des os des membres robustes. Durant les phases tempérées océaniques, l'expansion des forêts plus denses et la concurrence pour des ressources plus rares et de moindre qualité nutritive, affectent la taille des chevaux qui sont souvent plus petits. A contrario, les chevaux des périodes glaciaires peuvent être aussi caractérisés par leur petite taille en raison des conditions climatiques sévères associées à une faible productivité (biomasse végétale).

Il apparaît donc difficile d'établir un modèle général de la variation de la taille des chevaux au cours Pléistocène moyen sans tenir compte des variations d'ordre géogra- 


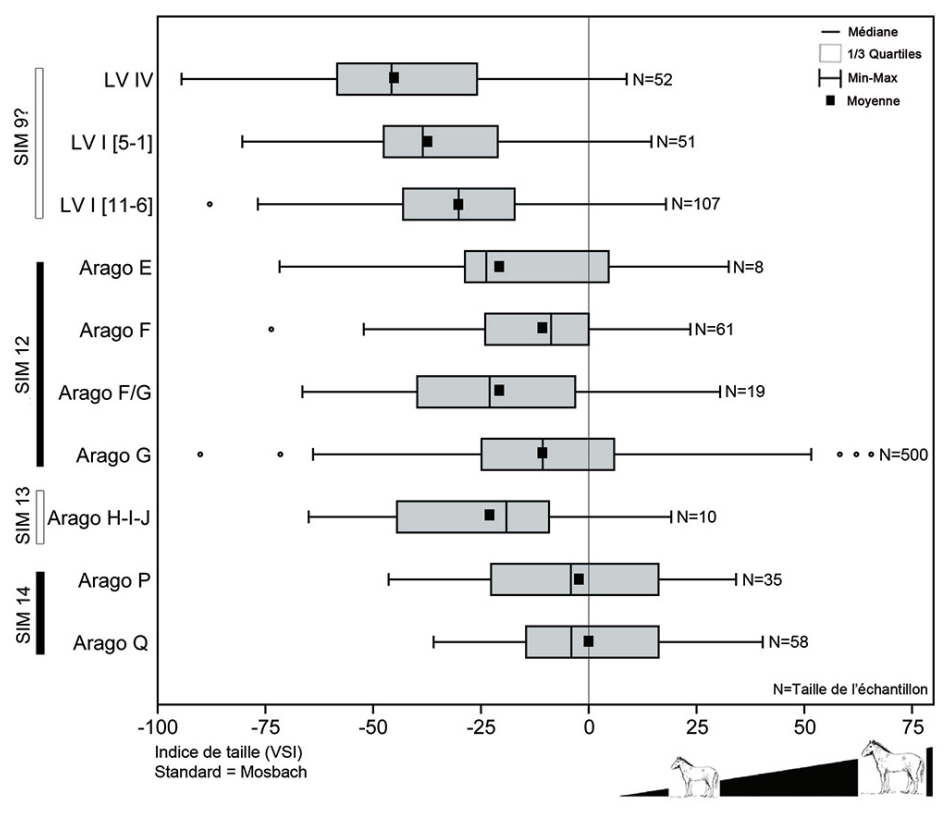

Figure 2 - Variation des indices de taille (VSI) des chevaux du complexe moyen de la Caune de l'Arago et de Lunel-Viel. Site standard : Mosbach [57] ; Caune de l'Arago [71]; Lunel-Viel (Eisenmann, com. pers.).

phiques et des caractéristiques intrinsèques des cycles climatiques qui se sont succédés au cours de cette période.

\subsubsection{Pléistocène supérieur}

À la fin du Pléistocène moyen puis durant le Pléistocène supérieur et l'Holocène, les chevaux subissent une diminution de taille quasi constante [56] à l'origine d'ailleurs de la création de plusieurs sous-espèces chronologiques [52, 58]. Cette tendance est par exemple bien illustrée dans la séquence archéologique de la grotte Tournal (Aude, Figure 3) : la taille des chevaux diminue significativement entre les niveaux moustériens $\mathrm{B} / \mathrm{C}$ et $\mathrm{D}$ et le niveau aurignacien $\mathrm{E}$, puis se poursuit entre celui-ci et les couches $\mathrm{G}$ et $\mathrm{H}$ rattachées au Magdalénien moyen et supérieur. La Figure 3 montre également que les chevaux plus anciens des Ramandils (Aude, SIM 5?, env. 100000 ans) et du Portel-Ouest F (Arrière, SIM 4/3 ?) sont plus grands que ceux des niveaux moustériens B/C et D de Tournal (SIM 3, 30 000-60 000 ans). La taille du cheval de Vacquerolles (Gard, début SIM 2, 12 000-30 000 ans) s'intercale bien entre les séries du Paléolithique supérieur de Tournal.

La diminution de la taille corporelle au Pléistocène supérieur constituerait « a gradual process evidently tracking climatic change, but without speciation 》 [74]. Les explications à ce processus pourraient être attribuées à un pédomorphisme ou nanisme adaptatif [74-77]. Elle nécessite de maintenir des densités de population dans 


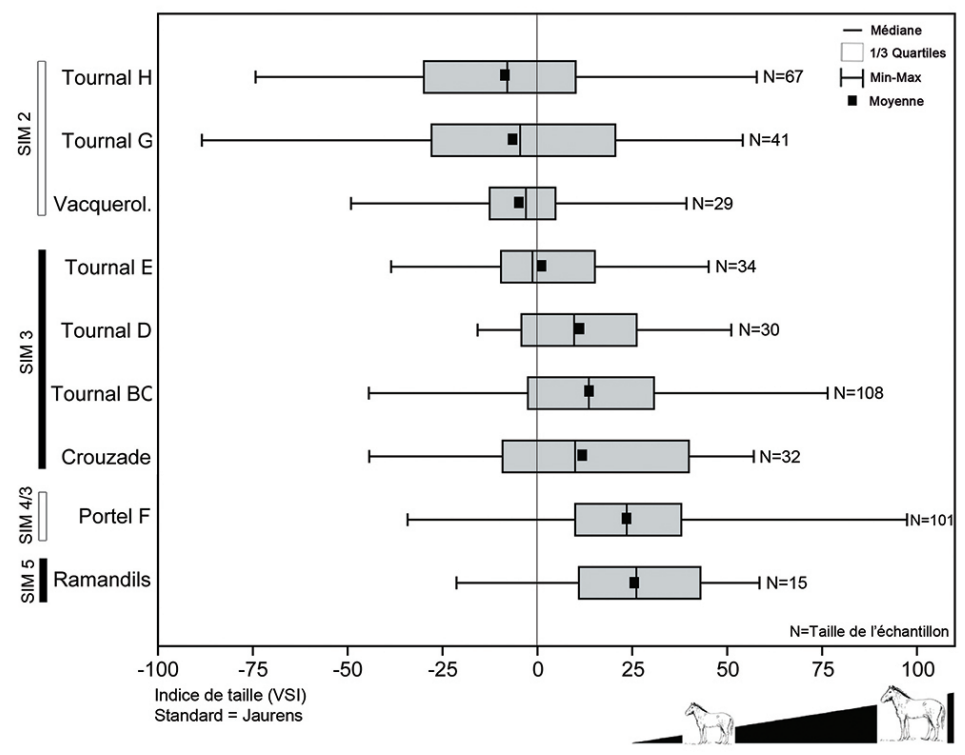

Figure 3 - Variation des indices de taille (VSI) des chevaux au Pléistocène supérieur. Site standard : Jaurens [78-79] ; Portel-Ouest F, Crouzade, Tournal [59] ; Ramandils, Vacquerolles (Boulbes, données pers.).

un environnement de plus en plus inégal ou contraignant, avec une stratégie visant à augmenter le taux de croissance des populations (réduction de la période de gestation par exemple) pour la survie de l'espèce [55]. Ceci pourrait constituer une réponse adaptative aux variations rapides du climat au cours de cette période [59]; la réduction de la taille assure dans ce cas un marqueur biochronologique intéressant.

Parallèlement à cette diminution de la taille corporelle, il est aussi possible de détecter des variations de certaines proportions squelettiques. Le graphique de la Figure 4 montre que les métacarpes des chevaux de la fin du Pléistocène moyen et du Pléistocène supérieur se classent chronologiquement en fonction de leur longueur moyenne mais un tri s'opère également le long de l'axe des ordonnées d'après la robustesse de la diaphyse. D'après la répartition des espèces actuelles, la robustesse serait bien corrélée aux conditions hygrométriques du milieu [80]. Les métacarpes des niveaux moustériens de Tournal B-D présentent par exemple une diaphyse relativement robuste (Figure 4), ce qui corrobore la forte humidité relevée dans ces niveaux [59]. Ces variations rapides et réversibles des proportions de la diaphyse semblent dans cette situation relever de la plasticité phénotypique plutôt que d'un mécanisme micro-évolutif.

\subsubsection{La fin du Pléistocène (Magdalénien)}

Les indices de taille reportés sur le graphique de la figure 5 montre une diminution progressive de la taille corporelle des chevaux du Sud de la France au Nord de l'Europe au Magdalénien. Les jalons de ce cline géographique sont bien visibles et les séries provenant des mêmes latitudes possèdent un gabarit équivalent. Dans le détail, les 


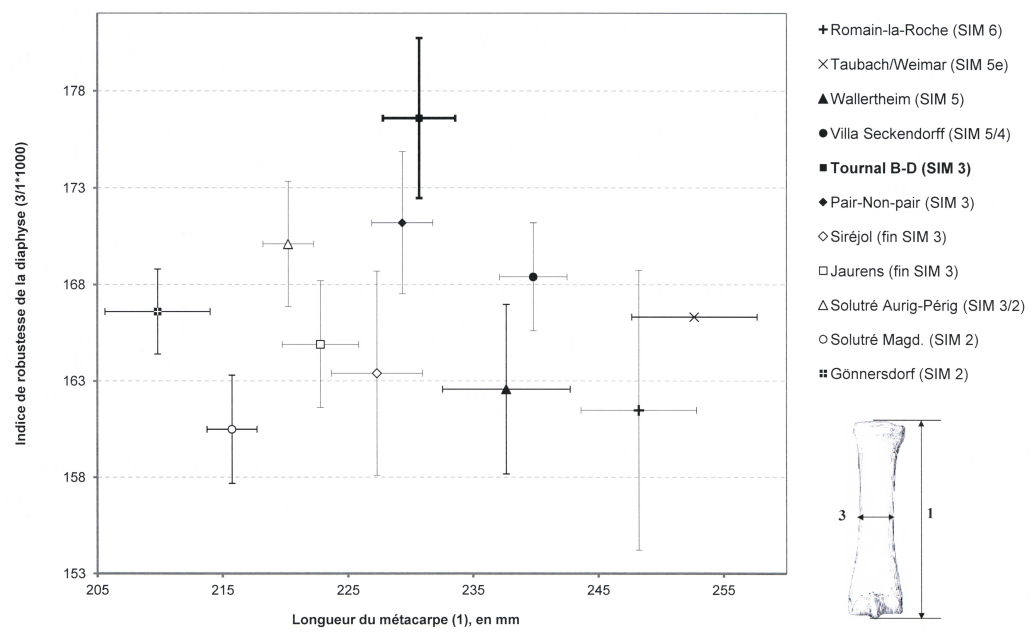

Figure 4 - Indice de robustesse moyen en fonction de la longueur moyenne des métacarpes de chevaux pléistocènes (SIM 6 à 2). Les barres d'erreur représentent l'intervalle de confiance (95\%). Romain-la-Roche, Tournal (Boulbes, données pers.); Taubach / Weimar (Einsenmann, comm. pers.); Wallertheim [53]; Villa Seckendorff, Gönnersdorf [57]; Pair-non-Pair [52]; Solutré [58] ; Siréjol, Jaurens [78]. Indice de robustesse = largeur de la diaphyse au milieu / longueur $* 1000$.

chevaux méditerranéens des niveaux $\mathrm{G}$ et $\mathrm{H}$ de Tournal sont en effet un peu plus grands que ceux de Saint-Germain-la-Rivière (Gironde) et du Quéroy I (Charente), eux même plus robustes que les chevaux du Centre de la France de Solutré (Saôneet-Loire) et du Rocs-aux-Sorciers (Vienne). Ces derniers désignent enfin des formes au gabarit plus important que celui des chevaux du bassin parisien du Grand Canton (Seine-et-Marne) et de Rhénanie (Gönnersdorf et Andernach).

Ces différences régionales peuvent être mises en parallèle avec les gradients de teneurs en azote $15 \mathrm{~N}$ et s'expliqueraient par une apparition plus précoce d'amélioration climatique dans le Sud par rapport aux régions du Nord et / ou continentale après le dernier maximum glaciaire [81-83]. L'accès à plus de ressources dans les régions plus méridionales aurait favorisé le maintien d'une taille égale dans le Sud de la France. Ces résultats traduisent une nouvelle fois la mosaïque régionale des populations de chevaux au Tardiglaciaire [57, 84-86]. 


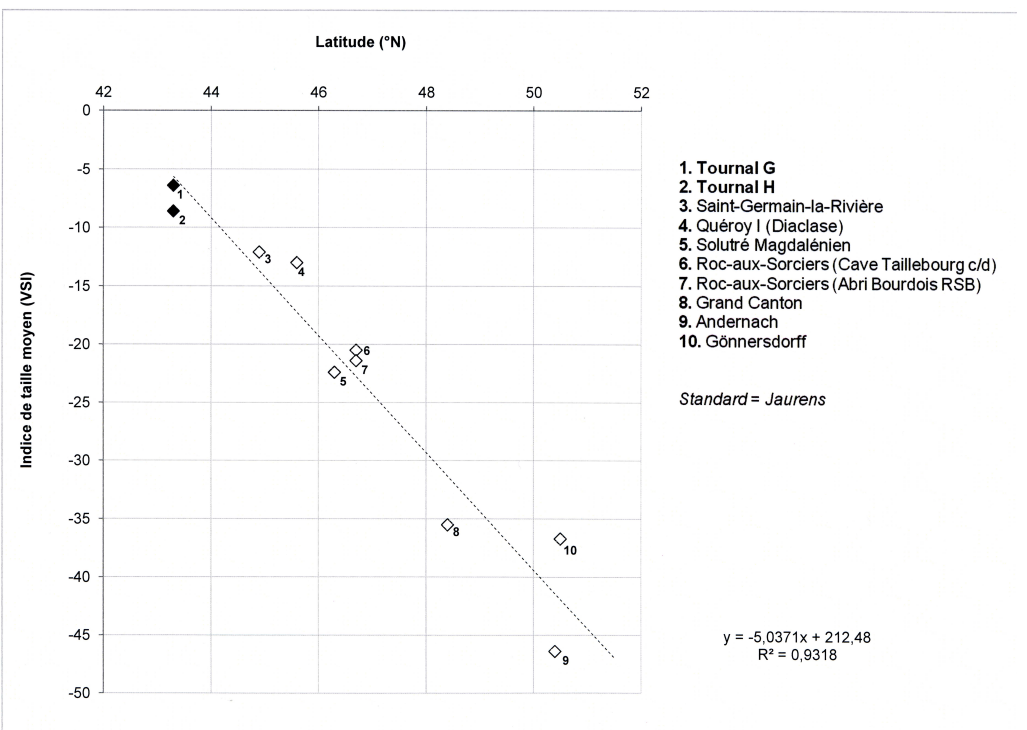

Figure 5 - Variation des indices de taille (VSI) des chevaux au Magdaléniens en fonction de la latitude. Site standard : Jaurens [78-79]; données compilées d'après [86]; Tournal [59]; Saint-Germain-la-Rivière [52]; Quéroy, Grand Canton (Eisenmann, com. pers.) ; Solutré [58] ; Rocs-aux-Sorciers [86]; Gönnersdorf, Andernach [57].

\subsection{Les bovinés (J.-Ph. B.)}

Les grands bovidés sont principalement représentés durant le Quaternaire par deux genres (ou sous-genres), Bos ou aurochs et Bison avec plusieurs espèces. Faisant suite au stock de bison archaïque du Pléistocène inférieur (Eobison), les vrais bisons évoluent en Eurasie, avec B. schoetensaki, le plus ancien, puis B. priscus le bison des steppes et $B$. bonasus le bison européen ou wisent, encore vivant de nos jours dans les forêts de Pologne et Lituanie (Bialowieza) [87-88]. Le bison des steppes est un animal grégaire de grande taille avec une forte encornure, adapté aux grandes plaines et pouvant supporter des froids rigoureux; alors que la forme actuelle est de plus petite taille, tant corporelle que socio-écologique (groupe social), avec des cornes plus réduites, pouvant vivre dans des environnements plus forestiers et peut être plus tempérés. Le premier, avec un port de tête plus bas, est typique des paisseurs ( «grazer $)$ ) alors que le second a une alimentation plus composite ( « mixed-feeder »). Leur morphologie externe reste globalement assez comparable (différence de la ligne dorsale et massivité de l'avant-train) et ils accusent, l'un comme l'autre, et comme de nombreux bovidés, un fort dimorphisme sexuel; le mâle étant de plus grande taille que la femelle, observable sur la quasi-totalité des éléments squelettiques. Il en va de même pour l'aurochs qui est une espèce polymorphe, de grande taille, avec 
des membres longs lui donnant une allure élancée et un cornage imposant avec une forte courbure. Il s'agit d'une espèce tempérée favorisant les milieux d'écotones et les prairies; ils subsistent pendant les phases glaciaires de l'Hémisphère nord dans les contrées plus méridionales, du Bassin méditerranéen.

Les bisons ont une vaste distribution spatio-temporelle, couvrant l'Eurasie (zone paléarctique) jusqu'en Amérique du Nord (néarctique) depuis le Pléistocène moyen (début à 780000 ans). L'aurochs a une moindre extension, en Eurasie, et ne dépasse pas le $60^{\circ} \mathrm{N}$ (et ne colonise pas l'Amérique). Il est présent aussi en Afrique du Nord et au Proche- et au Moyen-Orient.

Ce sont ces espèces et ces formes qui ont occupé l'Europe de l'Ouest et au-delà, et que les premiers groupes humains ont rencontré de manière très régulière tout au long du Paléolithique moyen à supérieur, Néandertalien à Sapiens. Avec les équidés et les cervidés ( $c f$. supra), ils représentent les proies préférentielles des différentes cultures se succédant; ils ont joué un rôle majeur dans les sociétés indiennes nord-américaines à l'Holocène (voir [89]) lorsque les troupeaux de bison étaient alors les ongulés les plus abondants jamais observés sur Terre (en dizaine de millions d'individus). Plusieurs gisements en Europe de l'Ouest démontre l'importance de ces ressources pour les groupes néandertaliens : par exemple les sites de Mauran et de Coudoulous I en France [90-93], de Wallertheim en Allemagne [94], mais aussi en Russie ou en Ukraine pour les Hommes modernes [89], avec plusieurs centaines d'individus accumulés dans des sites de boucherie et de consommation ( «kill-butchery sites ») parfois associés à des campements semi-permanents. Au Paléolithique supérieur, les grands bovidés jouent également un grand rôle dans les représentations symboliques et/ou artistiques (sculpture, gravure, peinture). Après la dernière glaciation, l'Aurochs devient un gibier de choix, souvent chassé et vénéré dans les temps protohistoriques à historiques.

\subsection{1 Évolution et taille corporelle}

Après ce cadre général, taxinomique, chronologique et paléoécologique, il est possible de retenir à la fois la grande différence entre sexes et l'importance cynégétique pour des groupes de chasseurs-cueilleurs. Les implications des variations de taille corporelle des populations à travers le temps sont d'ordre évolutif, écosystémique, mais aussi taphonomique et archéozoologique.

Il existe des différences significatives sur la taille en relation avec l'âge de l'animal, son sexe et les conditions environnementales. Aux États-Unis, les tailles moyennes varient entre les troupeaux de B. bison de différentes régions, et le poids des individus est souvent lié aux conditions de précipitation et de température; le facteur saisonnier est déterminant (Figure 6). Cela entraine des variations dans la production et la distribution des ressources animales (poids vif; viande, graisse, moelle). Les taux de croissance sont distincts entre populations et entre les sexes : plus rapides chez les femelles (plateau atteint entre 4-5 ans) alors qu'il est plus lent chez les mâles (env. 6 ans ou plus). De plus, les mâles ont des plus fortes proportions de l'avanttrain à l'inverse des femelles plus lourdes des arrière-trains [95]. Ces morphofonctions peuvent se répercuter sur les traits métriques des os (longueur, épaisseur corticale, âge de fusion des épiphyses). 


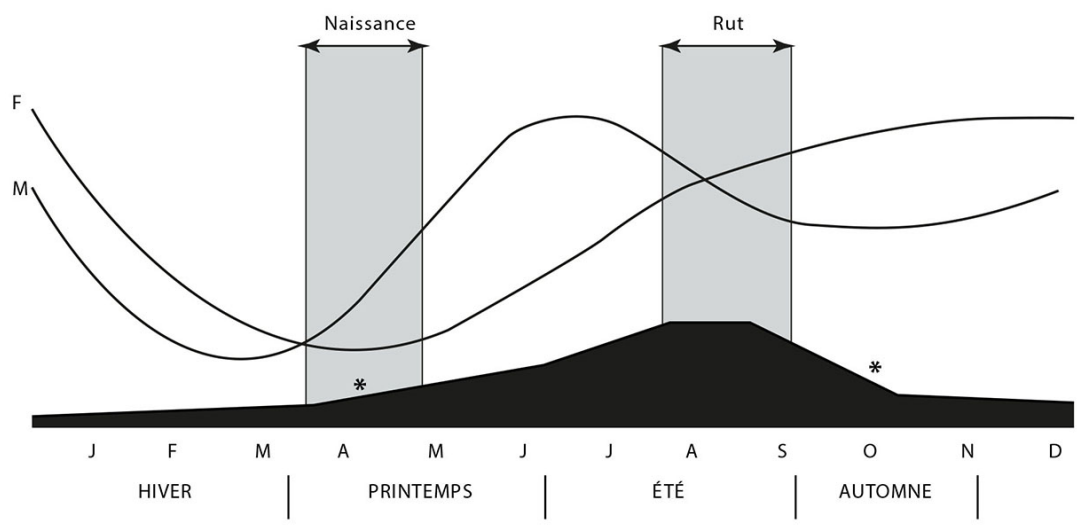

Figure 6 - Courbe de variation saisonnière théorique des poids chez des bisons adultes ( $\mathrm{M}=$ mâles $; \mathrm{F}=$ femelles $)$. Zone sombre en bas : périodes de reproduction et concentration des individus, avec $*=$ migrations / déplacements (in [92]).

Toutes ces variations ont des répercussions sur les ossements. La croissance étant variable, dans le cas d'accumulation fossile, l'action post-enfouissement et diagénétique va différer; la structure biologique (âge, sexe) contribuera ainsi au degré de conservation et représentation dans la documentation fossile. Les implications archéozoologiques concerneront alors la sélection des individus lors des chasses et le degré d'exploitation des carcasses, exprimé en termes de poids de viande et quantité de graisse, degré de fracturation osseuse ou de combustion.

Pour illustrer ces variations de taille, nous avons reporté les dimensions de longueur de métapodes, en particulier du métacarpien (Mcc), chez les bisons fossiles d'Europe de l'Ouest, distinguant les séries mâles des séries femelles d'un même gisement. Le Mcc est un élément assez diagnostic, abondant et retrouvé souvent complet, facile à sexer. Il existe des corrélations entre la longueur de ces os et la taille corporelle et des équations ont été calculées [96]; cependant ces équations sont générées sur des formes domestiques et nous préférons ici utiliser les valeurs biométriques brutes.

Treize gisements, principalement paléontologiques (seuls archéologiques : Mauran, Tournal) sont concernés (Figure 7), depuis ceux du Pléistocène moyen (Mosbach, Hundsheim, Châtillon-Saint-Jean, Coudoulous II), du début du Pléistocène supérieur (Roter-Berg), puis sept gisements datés des stades isotopiques 3 et 2 (soit entre 50000 et 10000 ans).

La tendance générale est une décroissance de la taille au cours du temps, bien marquée pour les séries du Pléistocène moyen au début du Pléistocène supérieur. Cela semble s'amortir durant le Pléistocène supérieur; mais, du fait d'une documentation plus abondante pour cette période relativement courte, nous observons des fluctuations. Elles rendent compte d'une grande variabilité spatio-temporelle des populations de bison contemporaines des hommes du Paléolithique supérieur (aurignacien, gravettien, solutréen, magdalénien). Aux processus évolutifs se rajoutent des facteurs écologiques, géotopographique et climatique, faisant varier les tailles et masses de ces animaux. 


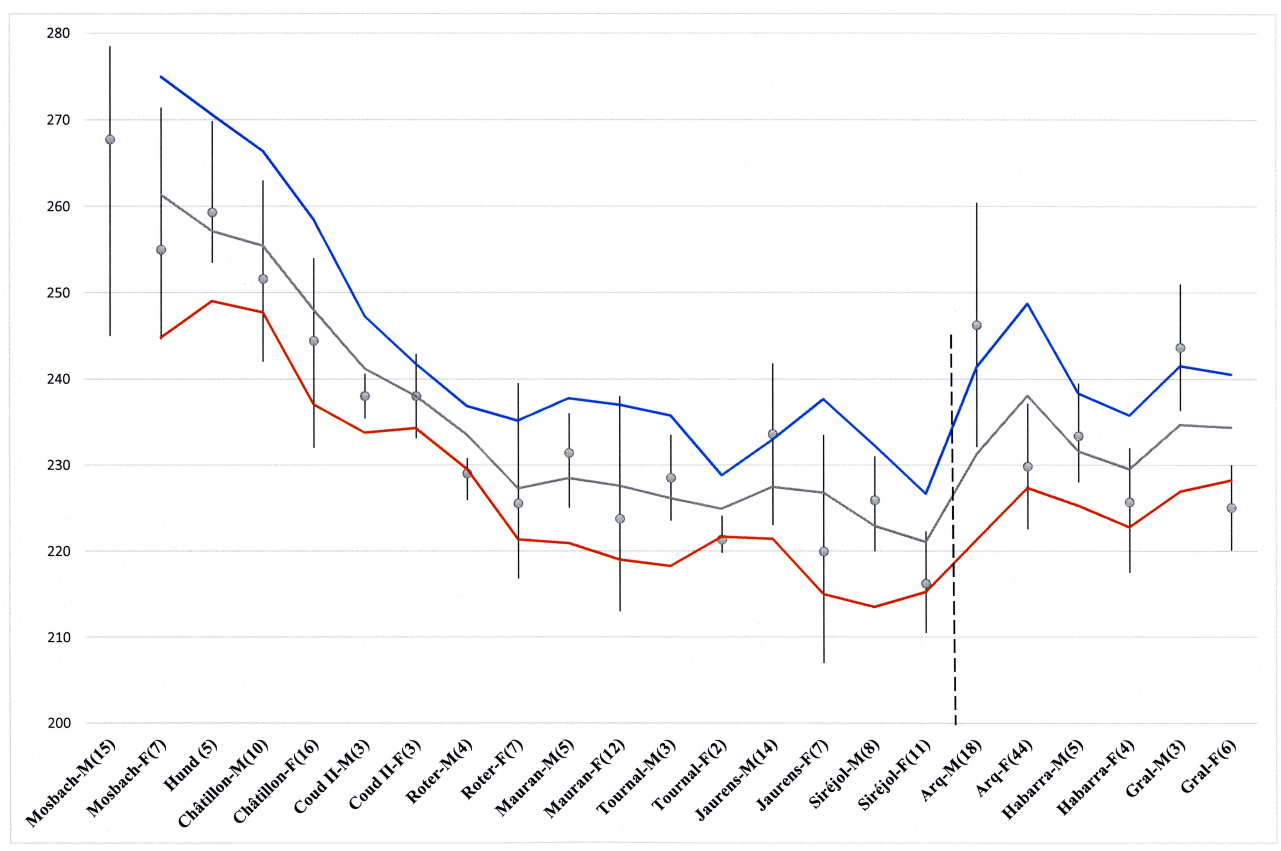

Figure 7 - Variation de la longueur du Métacarpien (min-moy-max, et n, en mm) de séries de bisons pléistocènes, selon les sexes $(\mathrm{M}=$ mâles ; $\mathrm{F}=$ femelles) ordonnée chronologiquement (le plus ancien à gauche). (Moyenne mobile de période 2 pour min-moy-max.)

De plus, des migrations de populations venant de l'Est peuvent encore compliquer ce schéma, en particulier l'existence du bison européen (B. bonasus) entre 47000 34000 ans en Europe de l'Ouest, première vague de ces migrations [97]. L'analyse paléogénétique montre que les individus échantillonnés des sites les plus récents de notre séquence (Arquet et Gral), possèdent (avec le site d'Habarra) une signature « wisent ». Ces populations de la fin du dernier glaciaire montrent une taille légèrement supérieure (Figure 7) par rapport à leurs prédécesseurs qui désignent le bison des steppes. De fait, les tailles entre les deux espèces ont parfois tendance à se confondre. Le schéma mis en évidence reste donc plus didactique que réel, car non exhaustif. L'intégration de nouvelles séries et un meilleur calage chronologique est nécessaire afin de mieux examiner les corrélations avec des courbes climatiques et détailler ces processus éco-évolutifs et biogéographiques dont les implications taphonomique et archéozoologiques sont essentielles.

\section{Conclusions}

Plusieurs modèles de variations sont mis en évidence chez les ongulés durant le Pléistocène illustrant des stratégies évolutives distinctes mais aussi des adaptations écomorphologiques importantes. Les variations dans les tailles corporelles des animaux, bien visibles à partir des dimensions des ossements, sont riches d'informations. La taille est corrélée à de nombreux facteurs ontogéniques, physiologiques mais égale- 
ment à des facteurs environnementaux tels que le climat (température, précipitation, humidité, saisonnalité) et les structures des biomes végétaux. À partir de l'étude de plusieurs membres de trois familles d'herbivores (cervidés, équidés, bovinés), plusieurs convergences se dégagent, partant d'un même substrat et des mêmes gisements, soit l'existence de formes de grande taille du Pléistocène moyen. Par la suite, on observe une tendance générale à la diminution de la taille corporelle des séries et populations fossiles de la fin du Pléistocène moyen et du Pléistocène supérieur. Il s'agit d'un processus évolutif assez général en Europe de l'Ouest, observable dans d'autres groupes de grands mammifères, qui a certainement impacté les activités cynégétiques des groupes humains. Dans un contexte anthropique, la taille et condition des herbivores-proies vont conditionner non seulement leurs stratégies d'acquisition (sélection des individus) mais aussi le degré de consommation et traitement des produits animaux (viande, os, moelle, peau...).

À ce premier facteur temporel s'ajoute des variations géographiques et saisonnières qui agissent à plus petite échelle, et sont plus étroitement liées aux conditions locales et à la variabilité des paramètres écologiques des milieux. Pour les espèces dimorphiques, les études doivent se diriger sur les différentes réponses apportées aux changements suivant les sexes et leurs stratégies de reproduction. La grande distribution spatiale des espèces telles que renne, cheval ou bison en Eurasie entraine une grande diversité et variabilité des populations au cours du Pléistocène, confirmant l'importance biogéographique des approches en paléoécologie et en taphonomie.

L'origine et la conservation des accumulations fossiles autant que l'impact des tailles des gibiers dans leurs acquisitions par des prédateurs, non-humains et humains, sont des éléments taphonomiques déterminants. Il est essentiel de bien identifier la structure paléobiologique des séries fossiles (taille, sex-ratio, âge, saisons) en relation avec nombreux facteurs intrinsèques (physiologie, etc.) et extrinsèques (géographie, végétation, climat) permettant également de mieux distinguer les processus micro-évolutifs (génétique) des réponses plastiques (phénotype).

\section{Remerciements}

N. B. remercie vivement V. Eisenmann pour ses données personnelles concernant les chevaux des gisements de Lunel-Viel, Grand Canton et Le Quéroy.

\section{Références}

[1] L. Blueweiss, H. Fox, V. Kudzma, D. Nakashima, R. Peters, S. Sams, Relationships between body size and some life history parameters. Oecologia. 37, 257-272, 1978.

[2] R.H. Peters, The ecological implication of body size, Cambridge University Press, Cambridge, 329 pages, 1983.

[3] J. Damuth, B.J. MacFadden, Body size in mammalian paleobiology : estimation and biological implication, Cambridge University Press, 397 pages, 1990.

[4] W.A. Calder III, Size, function, and life history, Dover publications, New-York, 431 pages, 1996.

[5] M. Marchini, L.M. Sparrow, M.N. Cosman, A. Dowhanik, C.B. Krueger, B. Hallgrimsson, C. Rolian, Impact of genetic correlation on the independent evolution of body mass and skeletal size in mammals. BMC Evolutionary Biology. 14 :258, DOI : 10.1186/s12862-014-0258-0, 2014.

[6] J. Damuth, Population density and body size in mammals. Nature, 290, 699-700, 1981. 
[7] R.H. Peters, K. Wassenberg, The effect of body size on animal abundance. Oecologia. 60, 89-96, 1983.

[8] W.D. Lambert, C.S. Holling, Causes of ecosystem transformation at the end of the Pleistocene : evidence from mammal body-mass distributions. Ecosystems. 1, 157-175, 1998.

[9] P.A. Marquet, M.L. Taper, On size and area : Patterns of mammalian body size extremes across landmasses. Evolutionary Ecology. 12, 127-139, 1998.

[10] R.G. Klein, K. Scott, Glacial/Interglacial size variation in fossil Spotted Hyenas (Crocuta crocuta) from Britain. Quaternary Research. 32, 88-95, 1989.

[11] M. Boudadi-Maligne, «Les Canis pléistocènes du sud de la France : approche biosystématique, évolutive et biochronologique », thèse de doctorat de l'Université de Bordeaux I, 446 pages, 2010.

[12] J.P. Brugal, M. Boudadi-Maligne, Quaternary small to large canids in Europe : taxonomic status and biochronological contribution. Quaternary International. 243, 171-182, 2011.

[13] J. Van der Made, K. Stefaniak, A. Marciszak, The Polish fossil record of the wolf Canis and deer Alces, Capreolus, Megaloceros, Dama and Cervus in an evolutionary perspective. Quaternary International. 326/327, 406-430, 2014.

[14] G. Sansalone, D.F. Bertè, L. Maiorino, L. Pandolfi, Evolutionary trends and statis in carnassial teeth of european Pleistocene wolf Canis lupus (Mammalia, Canidae). Quaternary Science Reviews. 110, 36-48, 2015.

[15] A.M. Lister, Evolutionary and ecological origins of British deer. Proceedings of the Royal Society of Edinburgh. 82, 205-229, 1984.

[16] J. Weinstock, « Late Pleistocene reindeer population in Middle and Western Europe. An osteometrical study of Rangifer tanrandus », thèse de doctorat de l'Université de Tübingen, Mo-Vince Verlag, 307 pages, 2000 .

[17] G. Di Stephano, L. Pandolfi, C. Petronio, L. Salari, Considerations on the morphometry and the occurrence of Cervus elaphus (Mammalia, Cervidae) from the Late Pleistocene of the Italian Peninsula. Rivista Italiana di Paleontologia e Stratigraphia. 121, 1-18, 2015.

[18] L. Pandolfi, L. Maiorino, G. Sansalone, Did the Late Pleistocene climatic changes influence evolutionary trends in body size of red deer? The study case of the Italian Peninsula. Palaeogeography, Palaeoclimatology, Palaeoecology. 440, 110-115, 2015.

[19] V. Eisenmann, Les chevaux quaternaires européens (Mammalia, Perissodactyla). Taille, typologie, biostratigraphie et taxonomie. Géobios. 24 (6), 747-759, 1991.

[20] M.T. Alberdi, J.L. Prado, E. Ortiz-Jaureguizar, Patterns of body size changes in fossil and living Equini (Perissodactyla). Biological Journal of the Linnean Society. 54, 349-370, 1995.

[21] L. Pandolfi, C. Petronio, L. Salari, Bos primigenius Bojanus, 1827 from the early Late Pleistocene deposit of Avetrana (Southern Italy) and the variation in size of the species in Southern Europe : preliminary report. Journal of Geological Research. 2011, 1-11, 2011.

[22] E. Wright, S. Viner-Daniels, Geographical variation in the size and shape of the European aurochs (Bos primigenius). Journal of Archaeological Science. 54, 8-22, 2015.

[23] C. Bergmann, Ueber die Verhältnisse der Wärmeökonomie der Thiere zu ihrer Größe. Göttinger Studien. 3, 595-798, 1847.

[24] J. Merilä, A.P. Hendry, Climate change, adaptation and phenotypic plasticity : the problem and the evidence. Evolutionary Applications. 7, 1-14, 2014.

[25] A.M. Lister, The impact of Quaternary Ice Ages on mammalian evolution. Philosophical Transactions of the Royal Society of London, B 359, 221-241, 2004.

[26] A.W.F. Banfield, A revision of the Reindeer and Caribou, Genus Rangifer, National Museum of Canada, 177, 137 pages, 1961.

[27] V. Geist, Deer of the world. Their evolution, behaviour and ecology, Editions Stackpole Books, Swan Hill Press, England, 421 pages, 1999.

[28] D.R. Klein, The introduction, increase, and crash of reindeer on St. Matthew Island. Journal of Wildlife Management. $32(2), 350-367,1968$.

[29] D.C. Thomas, P. Everson, Geographic variation in caribou on the Canadian arctic islands. Canadian Journal of Zoology. 60, 2442-2454, 1982. 
[30] T. Skogland, The effects of density dependent resource limitation on size of wild reindeer. Oecologia. 60, 156-168, 1983.

[31] P. Valkenburg, T.H. Spraker, M.T. Hinkes, L.H. van Daele, R.W. Tobey, R.A. Sellers, Increases in body weight and nutritional status of transplanted Alaskan caribou. Rangifer. Sp. Issue 12, 133-138, 1998.

[32] R.B. Weladji, $\varnothing$. Holand, Global climate change and reindeer : effects of winter weather on the autumn weight and growth of calves. Oecologia. 136, 317-323, 2003.

[33] R.D. Kahlke, N. García, D.S. Kostopoulos, F. Lacombat, A.M. Lister, P. Mazza, V.V. Titov, Western Palaearctic palaeoenvironmental conditions during the Early and early Middle Pleistocene inferred from large mammal communities, and implications for hominin dispersal in Europe. Quaternary Science Reviews. 30 (11), 1368-1395, 2011.

[34] J. Bouchud, «Essai sur le renne et la climatologie du Paléolithique moyen et supérieur », thèse de doctorat de l'Université de Paris, 300 pages, 1966.

[35] F. Delpech, Les faunes du Paléolithique supérieur dans le Sud-ouest de la France, Cahier du Quaternaire 6, Editions du CNRS, Paris, 463 pages, 1983.

[36] P. Magniez, «Etude paléontologique des Artiodactyles de la grotte Tournal. Etude taphonomique, archéozoologique et paléoécologique des grands Mammifères dans leur cadre biostratigraphique et paléoenvironnemental », thèse de Doctorat de l'Université de Perpignan, 792 pages, 2010a.

[37] P. Magniez, Incidences des fluctuations climatiques sur la taille du Renne (Rangifer tarandus) au Pléistocène supérieur. Quaternaire. 21 (3), 259-279, $2010 \mathrm{~b}$.

[38] D. Kuntz, « Ostéométrie et migration(s) du Renne (Rangifer tarandus) dans le Sud-Ouest de la France au cours du dernier Pléniglaciaire et du Tardiglaciaire (21500-13000 Cal. BP) », thèse de Doctorat de l'Université de Toulouse II, 396 pages, 2011.

[39] P. Auguste, Les Cervidae (Mammalia, Cetartiodactyla) du gisement pléistocène moyen final de l'aven de Romain-la-Roche (Doubs, France). Revue de Paléobiologie Genève. 29 (2), 619-653, 2010.

[40] J.L. De Beaulieu, V. Andrieu, J.J. Lowe, P. Ponel, M. Reille, The Weichselian Late-glacial in southwestern Europe (Iberian Peninsula, Pyrenees, Massif Central, northern Apennines). Journal of Quaternary Science. 9, 101-107, 1994.

[41] T. Skogland, The effects of food and maternal conditions on fetal growth and size in wild reindeer. Rangifer. 4 (2), 39-46, 1984.

[42] T. Skogland, The effects of density-dependent resource limitations on the demography of wild reindeer. Journal of Animal Ecology. 54, 359-374, 1985.

[43] M. Garel, E.J. Soldberg, B.-E. Saether, I. Herfindal, K.-A. Høgda, The length of growing season and adult sex ratio affect sexual size dimorphism in moose. Ecology. 87 (3), 745-758, 2006.

[44] F. Rivals, M.A. Julien, M. Kuitems, T. Van Kolfschoten, J. Serangeli, D.G. Drucker, H. Bocherens, N.J. Conard, Investigation of equid paleodiet from Schoningen 13 II-4 through dental wear and isotopic analyses : Archaeological implications. Journal of Human Evolution. 129-137, 2015.

[45] D. Pushkina, H. Bocherens, R. Ziegler, Unexpected palaeoecological features of the Middle and Late Pleistocene large herbivores in southwestern Germany revealed by stable isotopic abundances in tooth enamel. Quaternary International. 339-340, 164-178, 2014.

[46] E.N. van Asperen, Ecomorphological adaptations to climate and substrate in late Middle Pleistocene caballoid horses. Palaeogeography, Palaeoclimatology, Palaeoecology. 297, 584-596, 2010a.

[47] M. Kuitems, J. van der Plicht, D.G. Drucker, T. van Kolfschoten, S.W.L. Palstra, H. Bocherens, Carbon and nitrogen stable isotopes of well-preserved Middle Pleistocene bone collagen from Schoningen (Germany) and their paleoecological implications. Journal of Human Evolution. 89, 105-113, 2015.

[48] B.J. Macfadden, Fossil Horses : Systematics, Paleobiology and Evolution of the Family Equidae, Cambridge University Press, 384 pages, 1994.

[49] E.N. van Asperen, Implications of age variation and sexual dimorphism in modern equids for Middle Pleistocene equid taxonomy. International Journal of Osteoarchaeology. 23 (1), 1-12, $2010 \mathrm{~b}$.

[50] V. Gromova, Histoire des chevaux (genre Equus) de l'ancien monde (Première partie) : Revue et description des formes, Travaux de l'Institut de Paléontologie de l'Académie des Sciences U.R.S.S., 17 (1), 373 pages. Trad. Pietresson de Saint-Aubin, 1955. Annales du Centre d'études et de Documentation paléontologique 13, 1949. 
[51] O. Sickenberg, Uber die Grösse der pleistozänen Pferde der caballus-Gruppe in Europa und Nordasien. Eiszeitalter und Gegenwart. 12, 99-124, 1962.

[52] F. Prat, « Recherche sur les Equidés pléistocènes en France », thèse de Doctorat de l'Université de Bordeaux, 696 pages, 1968.

[53] G. Nobis, Vom Wildpferd zum Hauspferd. Fundamenta, Monographien zur Urgeschichte, Reihe B, 6, Bauhlau Verlag, Köln, 96 pages, 1971.

[54] A. Forsten, Size decrease in Pleistocene-Holocene true or caballoid horses of Europe. Mammalia. 55, 407-419, 1991.

[55] A. Forsten, Size decrease in Late Pleistocene-Holocene caballoid horses (Genus Equus) intra- or interspecific evolution? A discussion of alternatives. Quaternary International. 19, 71-75, 1993.

[56] V. Eisenmann, F. David, «Evolution de la taille des Chevaux d'Arcy-sur-Cure et de quelques autres Chevaux quaternaires » dans L'Aurignacien de la Grotte du Renne, eds. B. Schmider, Gallia Préhistoire, Supplément 34, Editions du CNRS, Paris, p. 97-102, 2002.

[57] B. Cramer, « Morphometrische Untersuchungen an Quartären Pferden in Mitteleuropa », thèse de doctorat de l'Université de Tübingen, 165 pages, 2002.

[58] J.L. Guadelli, Les chevaux de Solutré (Saône et Loire, France). Cahier du Quaternaire. 16, 261-336, 1991.

[59] P. Magniez, N. Boulbes, Environmental at the transition Middle and Late Paleolithic in South of France. The archeosequence of the Tournal cave (Bize-Minervois, France). Quaternary International. 337, 43-63, 2014.

[60] R.D. Guthrie, Rapid body size decline in Alaskan Pleistocene horses before extinction. Nature. 426, 169-171, 2003.

[61] H.P. Uerpmann, Faunal remains from Shams-ed-Din Tannira, a Halafian site in northern Syria. Berytus. 30, 3-52, 1982 .

[62] H. de Lumley, A. Fournier, Y.C. Park, Y. Yokoyama, A. Demouy, Stratigraphie du remplissage pléistocène moyen de la Caune de l'Arago. L'Anthropologie. 88, 5-18, 1984.

[63] A.M. Moigne, M.R. Palombo, V. Belda, D. Heriech-Briki, S. Kacimi, F. Lacombat, M.A. de Lumley, J. Moutoussamy, F. Rivals, J. Quiles, A. Testu, Les faunes de grands mammifères de la Caune de l'Arago (Tautavel) dans le cadre biochronologique des faunes du Pléistocène moyen italien. L'Anthropologie. 110, 788-831, 2006.

[64] C. Hanquet, E. Desclaux, Analyse paléoécologique des communautés de micromammifères de la Caune de l'Arago (Tautavel, France) dans le contexte des migrations de faunes en Europe méridionale au cours du Pléistocène Moyen. Quaternaire. 22 (1), 35-45, 2011.

[65] C. Perrenoud, C. Falguères, A.M. Moigne, A. Testu, P. Magniez, N. Boulbes, L. Lebreton, C. Hanquet, E. Desclaux, A.-S. Lartigot-Campin, V. Celiberti, S. Grégoire, H. de Lumley, C. Viallet, T. Chevalier, M.A. de Lumley, A. Vialet, C. Fontaneil, V. Pois, H.-M. Hu, C.-C. Shen, V. Michel, Diversité des occupations humaines en contexte glaciaire à la Caune de l'Arago (Tautavel, Pyrénées Orientales, France). Colloque Q10 AFEQ - CNF INQUA, Bordeaux, 16-18 Février 2016, 2016.

[66] L. Lebreton, E. Desclaux, C. Hanquet, A.M. Moigne, C. Perrenoud, Environmental context of the Caune de l'Arago Acheulean occupations (Tautavel, France), new insights from microvertebrates in Q-R levels, Quaternary International. 411, B, 182-192, 2016.

[67] J. Renault-Miskovsky, « Vegetación y Paleoclimatología del Pleistoceno Medio en la cuenca mediterránea francesa » dans Evolución humana en Europa y los yacimientos de la Sierra de Atapuerca, eds. J.M. Bermudez de Castro Risueño, E. Carbonell i Roura, J.L. Arsuaga, Editions Junta de Castilla y León, 1, p. 75-98, 1995.

[68] F. Rivals, E. Schulz, T.M. Kaiser, Climate related dietary diversity of the ungulate faunas from the Middle Pleistocene succession (OIS 14-12) at the Caune de l'Arago (France). Paleobiology. 38, 117-127, 2008.

[69] M.F. Bonifay, Le Cheval du Pléistocène moyen des grottes de Lunel-Viel (Hérault). Gallia Préhistoire. 23 (2), 233-281, 1980.

[70] V. Eisenmann, E. Crégut-Bonnoure, A.M. Moigne, Equus mosbachensis et les grands chevaux de la Caune de l'Arago et de Lunel-Viel : craniologie comparée. Bulletin du Muséum National d'Histoire Naturelle de Paris. 4 (7), 17-173, 1985. 
[71] N. Boulbes, «Etude préliminaire du cheval de l'unité archéostratigraphique Q », dans Caune de l'Arago (Tautavel, Pyrénées-Orientales), Rapport de fouille 2014, dir. C. Perrenoud, SRA-DRAC LanguedocRoussillon, pages 48-55, 2014.

[72] E. Stephan, Sauerstoffisotopenverhältnisse im Knochengewebe grosser terrestrischer Säugetiere, Tübinger geowissenschaftliche Arbeiten : Reihe E, Mineralogie, Petrologie und Geochemie Mineralogie, Petrologie und Geochemie, 6, eds. Shaker, 218 pages, 1999

[73] E.N. van Asperen, Late Middle Pleistocene horse fossils from northwestern Europe as biostratigraphic indicators. Journal of Archaeological Science. 39 (7), 1974-1983, 2012.

[74] A. Forsten, The Pleistocene horses from Achenheim, near Strasbourg (Alsace, France), and a comparison with contemporaneous finds. Neues Jahrbuch für Geologie und Paläontologie, Abhandlungen. 201 (2), 163-177, 1996.

[75] S.J. Gould, « Palaeontology plus ecology as paleobiology », dans Theoretical ecology : principles and applications, eds. R.M. May, Blackwell Scientific Publications, London, pages 218-236, 1976.

[76] R.D. Guthrie, « Mosaics, allelochemics and nutrients, an ecological theory of Late Pleistocene megafaunal extinctions », dans Quaternary Extinctions, a Prehistoric Revolution, eds. P.S. Martin, R.G. Klein, University of Arizona Press, Tuscon, pages 259-298, 1984.

[77] A. Forsten, Middle Pleistocene replacement of stenonid horses by caballoid horses. Ecological implications. Paleogeography, Paleoclimatology, Paleoecology. 65, 23-33, 1988.

[78] V. Eisenmann, Website 2016. http ://www.vera-eisenmann.com. Access December, 2016.

[79] C. Mourer-Chauvirer, Le gisement Pléistocène supérieur de la grotte de Jaurens à Nespouls (Corrèze, France) : les Equidés (Mammalia, Perissodactyla). Nouvelles Archives du Muséum d'Histoire Naturelle de Lyon. 18, 17-60, 1980.

[80] V. Eisenmann, Sur quelques caractères adaptatifs du squelette d'Equus et leurs implications paléoécologiques. Bulletin du Muséum National d'Histoire Naturelle. 4ème série, 6, section C, 2, 185-195, 1984.

[81] D.G. Drucker, H. Bocherens, D. Biliou, Evidence for shifting environmental conditions in Southwestern France from 33000 to 15000 years ago derived from carbon-13 and nitrogen-15 abundances in collagen of large herbivores. Earth and Planetary Science Letters. 216, 163-173, 2003.

[82] R.E. Stevens, R.E.M. Hedges, Carbon and nitrogen stable isotope analysis of northwest European horse bone and tooth collagen, 40,000 BP-present : Palaeoclimatic interpretations. Quaternary Science Review. 23, 977-991, 2004.

[83] R.E. Stevens, T.C. O'Connell, R.E. Hedges, M. Street, Radiocarbon and stable isotope investigations at the Central Rhineland sites of Gönnersdorf and Andernach-Martinsberg, Germany. Journal of Human Evolution. 57 (2), 131-148, 2009.

[84] L.M. Kaagan, « The horse in late Pleistocene and Holocene Britain », thèse de doctorat de l'Université de Londres, 432 pages, 2000.

[85] O. Bignon, M. Baylac, J.D. Vigne, V. Eisenmann, Geometric morphometrics and the population diversity of Late Glacial horses in Western Europe (Equus caballus arcelini) : phylogeographic and anthropological implications. Journal of Archaelogical Science. 32, 375-391, 2005.

[86] P. Valensi, N. Boulbes, «La faune magdalénienne du Roc-aux-Sorciers (Angles-sur-l'Anglin, Vienne), Collection Suzanne de Saint-Mathurin », dans Angles-sur-l'Anglin (Vienne). L'abri-sous-roche du Roc-auxSorciers, ses occupants les Magdaléniens et leur rapport à l'art, dir.G. Pinçon, en préparation.

[87] V.E. Sokolov (dir.), European Bison -Morphology, systematics, evolution, ecology (en russe), Moscou, Nauka, (USSR Ac. of Sc.), 495 pages. 1979.

[88] C.C. Flerov, «Systematics and Evolution », dans European Bison -Morphology, systematics, evolution, ecology (en russe), dir. V. E. Sokolov, Moscou, Nauka, pages 9-127 (USSR Ac. of Sc.), 1979.

[89] J.P. Brugal, F. David, J. Enloe, J. Jaubert (dir.), Le Bison : gibier et moyen de subsistance des hommes du Paléolithique aux Paléoindiens des grandes plaines, Editions APDCA, Antibes, 562 p. (Actes du Colloque international, Toulouse, 6-10 juin 1995), 1999

[90] C. Farizy, F. David, J. Jaubert, Hommes et Bisons au Pléolithique moyen à Mauran (Haute-Garonne), Suppl. Gallia Préhistoire XXX, Editions du CNRS, Paris, 259 pages, 1994.

[91] J. Jaubert, B. Kervazo, J.P. Brugal, C. Falgueres, M. Jeannet, A. Louchard, H. Martin, F. Maksud, V. Mourre, Y. Quinif, «La séquence Pléistocène moyen de Coudoulous I (Lot). Bilan pluridisciplinaire », dans Les premiers peuplements en Europe : Données récentes sur les modalités de peuplement et sur le cadre chronostratigraphique, géologique et paléogéographique des industries du Paléolithique ancien et moyen en 
Europe, eds. N. Molines, M.H. Moncel, J.L. Monnier, Actes du Colloque international de Rennes, 22-25 septembre 2003, British Archaeological Reports, International Series 1364, Oxford, pages 237-251, 2005.

[92] J.P. Brugal, « Etude des populations de grands Bovidés européens : intérêt pour la connaissance des comportements humains au Paléolithique », dans Le Bison, gibier et moyen de subsistance des hommes du paléolithique aux paléoindiens des grandes plaines, dir. J.P. Brugal, F. David, J. Enloe, J. Jaubert, Editions APDCA, Antibes, pages 85-103, 1999a.

[93] J.P. Brugal, « Middle palaeolithic subsistence on large bovids : La Borde and Coudoulous I (Lot, France) », dans The role of early humans in the accumulation of European lower and middle palaeolithic bone assemblages, dir. S. Gaudzinski, E. Turner, Monographien des Römisch-Germanischen Zentralmuseums, Mainz, 42, pages 263-266, 1999b.

[94] S. Gaudzinski, On bovid assemblage and their consequence for the knowledge of subsistence patterns in Middle Palaeolithic. Proceedings of the Prehistoric Society. 62, 19-39, 1996.

[95] A.M. Emerson, « Archaeological implications of variability in the economic anatomy of Bison bison », thèse de doctorat de l'Université de Washington State, 771 pages, 1990.

[96] J. Matolcsi, Historische Erforshung der Körpergrösse des Rindes auf Grund von ungarishem Knochenmaterial. Zeitschrift für Tierzürchtung und Züchtungsbiologie (Journal of Animal Breeding and Genetics). 87 (1-4), 89-137, 1970 .

[97] D. Massilani, S. Guimaraes, J.P. Brugal, E.A. Bennett, M. Tokarska, R.M. Arbogast, G. Baryshnikov, G. Boeskorov, J.C. Castel, S. Davydov, S. Madelaine, O. Putelat, N. Spasskaya, H.P. Uerpmann, T. Grange, E.M. Geigl, Past climate changes, population dynamics and the origin of Bison in Europe. BMC Biology. 14, 93, DOI 10.1186/s12915-016-0317-7, 2016. 